PROCEEDINGS OF THE

AMERICAN MATHEMATICAL SOCIETY

Volume 135, Number 9, September 2007, Pages 2707-2711

S 0002-9939(07)08805-3

Article electronically published on May 2, 2007

\title{
A SMOOTH COUNTEREXAMPLE TO NORI'S CONJECTURE ON THE FUNDAMENTAL GROUP SCHEME
}

\author{
CHRISTIAN PAULY \\ (Communicated by Ted Chinburg)
}

\begin{abstract}
We show that Nori's fundamental group scheme $\pi(X, x)$ does not base change correctly under extension of the base field for certain smooth projective ordinary curves $X$ of genus 2 defined over a field of characteristic 2 .
\end{abstract}

\section{INTRODUCTION}

In the paper $[\mathrm{N}]$ Madhav Nori introduced the fundamental group scheme $\pi(X, x)$ for a reduced and connected scheme $X$ defined over an algebraically closed field $k$ as the Tannaka dual group of the Tannakian category of essentially finite vector bundles over $X$. In characteristic zero $\pi(X, x)$ coincides with the étale fundamental group, but in positive characteristic it does not (see, e.g., [MS]). By analogy with the étale fundamental group, Nori conjectured that $\pi(X, x)$ base changes correctly under extension of the base field. More precisely:

Nori's conjecture (see [MS, page 144 or $[\mathrm{N}$, page 89). If $K$ is an algebraically closed extension of $k$, then the canonical homomorphism

$$
h_{X, K}: \pi\left(X_{K}, x\right) \longrightarrow \pi(X, x) \times_{k} \operatorname{Spec}(K)
$$

is an isomorphism.

In [MS] V.B. Mehta and S. Subramanian show that Nori's conjecture is false for a projective curve with a cuspidal singularity. In this note (Corollary 4.2) we show that certain smooth projective ordinary curves of genus 2 defined over a field of characteristic 2 also provide counterexamples to Nori's conjecture.

The proof has two ingredients: the first is an equivalent statement of Nori's conjecture in terms of $F$-trivial bundles due to V.B. Mehta and S. Subramanian (see section 2), and the second is the description of the action of the Frobenius map on rank- 2 vector bundles over a smooth ordinary curve $X$ of genus 2 defined over a field of characteristic 2 (see section 3 ). In section 4 we explicitly determine the set of $F$-trivial bundles over $X$.

I would like to thank V.B. Mehta for introducing me to these questions and for helpful discussions.

Received by the editors January 10, 2006 and, in revised form, May 18, 2006.

2000 Mathematics Subject Classification. Primary 14H60, 14D20; Secondary 14H30.

(C)2007 American Mathematical Society Reverts to public domain 28 years from publication 2707 


\section{Nori's CONJECTURE AND $F$-TRIVIAL BUNDLES}

Let $X$ be a smooth projective curve defined over an algebraically closed field $k$ of characteristic $p>0$. Let $F: X \rightarrow X$ denote the absolute Frobenius of $X$ and $F^{n}$ its $n$-th iterate for some positive integer $n$.

2.1. Definition. A rank- $r$ vector bundle $E$ over $X$ is said to be $F^{n}$-trivial if

$$
E \text { is stable and } F^{n *} E \cong \mathcal{O}_{X}^{r} .
$$

2.2. Proposition (MS Proposition 3.1). If the canonical morphism $h_{X, K}$ (see (1.1)) is an isomorphism, then any $F^{n}$-trivial vector bundle $E_{K}$ over $X_{K}:=X \times_{k}$ $\operatorname{Spec}(K)$ is isomorphic to $E_{k} \otimes_{k} K$ for some $F^{n}$-trivial vector bundle $E_{k}$ over $X$.

\section{The ACtion of the Frobenius map on RANK-2 VeCtor BUndLes}

We briefly recall some results from [LP1] and [LP2].

Let $X$ be a smooth projective ordinary curve of genus 2 defined over an algebraically closed field $k$ of characteristic 2. By [LP2, section 2.3] the curve $X$ equipped with a level-2 structure can be uniquely represented by an affine equation of the form

$$
y^{2}+x(x+1) y=x(x+1)\left(a x^{3}+(a+b) x^{2}+c x+c\right),
$$

for some scalars $a, b, c \in k$. Let $\mathcal{M}_{X}$ denote the moduli space of $S$-equivalence classes of semistable rank-2 vector bundles with trivial determinant over $X$; see, e.g., LeP. We identify $\mathcal{M}_{X}$ with the projective space $\mathbb{P}^{3}$ (see [LP1] Proposition 5.1). We denote by $V: \mathbb{P}^{3} \rightarrow \mathbb{P}^{3}$ the rational map induced by pull-back under the absolute Frobenius $F: X \rightarrow X$. There are homogeneous coordinates $\left(x_{00}: x_{01}: x_{10}: x_{11}\right)$ on $\mathbb{P}^{3}$ such that the equations of $V$ are given as follows (see [LP2, section 5]):

$$
V\left(x_{00}: x_{01}: x_{10}: x_{11}\right)=\left(\sqrt{a b c} P_{00}^{2}(x): \sqrt{b} P_{01}^{2}(x): \sqrt{c} P_{10}^{2}(x): \sqrt{a} P_{11}^{2}(x)\right),
$$

with

$$
\begin{gathered}
P_{00}(x)=x_{00}^{2}+x_{01}^{2}+x_{10}^{2}+x_{11}^{2}, \quad P_{10}(x)=x_{00} x_{10}+x_{01} x_{11}, \\
P_{01}(x)=x_{00} x_{01}+x_{10} x_{11}, \quad P_{11}(x)=x_{00} x_{11}+x_{10} x_{01} .
\end{gathered}
$$

Given a semistable rank-2 vector bundle $E$ with trivial determinant, we denote by $[E] \in \mathcal{M}_{X}=\mathbb{P}^{3}$ its $S$-equivalence class. The semistable boundary of $\mathcal{M}_{X}$ equals the Kummer surface $\operatorname{Kum}_{X}$ of $X$. Given a degree 0 line bundle $N$ on $X$, we also denote the point $\left[N \oplus N^{-1}\right] \in \mathbb{P}^{3}$ by $N$.

3.1. Proposition ([LP1] Proposition 6.1 (4)). The preimage $V^{-1}(N)$ of the point $N \in \operatorname{Kum}_{X} \subset \mathcal{M}_{X}=\mathbb{P}^{3}$ with coordinates $\left(x_{00}: x_{01}: x_{10}: x_{11}\right)$

- is a projective line if $x_{00}=0$;

- consists of the 4 square roots of $N$ if $x_{00} \neq 0$.

\section{Computations}

In this section we prove the following.

4.1. Proposition. Let $X=X_{a, b, c}$ be the smooth projective ordinary curve of genus 2 given by the affine model (3.1). Suppose that

$$
a^{2}+b^{2}+c^{2}+a+c=0 .
$$


Then there exists a nontrivial family $\mathcal{E} \rightarrow X \times S$ parametrized by a 1-dimensional variety $S$ (defined over $k$ ) of $F^{4}$-trivial rank-2 vector bundles with trivial determinant over $X$. Moreover any $F^{4}$-trivial rank-2 vector bundle $E$ with trivial determinant appears in the family $\mathcal{E}$, i.e., is of the form $\left(\operatorname{id}_{X} \times s\right)^{*} \mathcal{E}$ for some $k$-valued point $s: \operatorname{Spec}(k) \rightarrow S$.

We therefore obtain a counterexample to Nori's conjecture.

4.2. Corollary. Let $X=X_{a, b, c}$ be a curve satisfying (4.1). Then for any algebraically closed extension $K$, the morphism $h_{X, K}$ is not an isomorphism.

Proof. Since $S$ is 1-dimensional, there exists a $K$-valued point $s: \operatorname{Spec}(K) \rightarrow S$ that is not a $k$-valued point. Then the bundle $E_{K}=\left(\operatorname{id}_{X} \times s\right)^{*} \mathcal{E}$ over $X_{K}$ is not of the form $E_{k} \otimes_{k} K$. Now apply Proposition 2.2.

Proof of Proposition 4.1. The method of the proof is to determine explicitly all $F^{n_{-}}$ trivial rank-2 vector bundles $E$ over $X$ for $n=1,2,3,4$. Taking tensor products of $E$ with $2^{n+1}$-torsion line bundles allows us to restrict attention to $F^{n}$-trivial vector bundles with trivial determinant.

We first compute the preimage under iterates of $V$ of the point $A_{0} \in \mathbb{P}^{3}$ determined by the trivial rank-2 vector bundle over $X$. We recall (see, e.g., [LP1, Lemma 2.11 (i)) that the coordinates of $A_{0} \in \mathbb{P}^{3}$ in the coordinate system $\left(x_{00}\right.$ : $\left.x_{01}: x_{10}: x_{11}\right)$ are $(1: 0: 0: 0)$. It follows from Proposition 3.1 and equations (3.2) that $V^{-1}\left(A_{0}\right)$ consists of the 4 points

$$
(1: 0: 0: 0),(0: 1: 0: 0),(0: 0: 1: 0) \text { and }(0: 0: 0: 1) \text {, }
$$

which correspond to the 2-torsion points of the Jacobian of $X$. Abusing notation we denote by $A_{1}$ both the 2 -torsion line bundle on $X$ and the point $(0: 1: 0: 0) \in \mathbb{P}^{3}$.

Both points $A_{0}$ and $A_{1}$ correspond to $S$-equivalence classes of semistable rank-2 vector bundles. The set of isomorphism classes represented by the two $S$-equivalence classes $A_{0}$ and $A_{1}$ equal $\mathbb{P} \operatorname{Ext}^{1}\left(A_{1}, A_{1}\right) \cup\{0\}$ and $\mathbb{P} \operatorname{Ext}^{1}\left(\mathcal{O}_{X}, \mathcal{O}_{X}\right) \cup\{0\}$, respectively, where 0 denotes the trivial extensions $A_{1} \oplus A_{1}$ and $\mathcal{O}_{X} \oplus \mathcal{O}_{X}$. Note that the two cohomology spaces $\operatorname{Ext}^{1}\left(A_{1}, A_{1}\right)$ and $\operatorname{Ext}^{1}\left(\mathcal{O}_{X}, \mathcal{O}_{X}\right)$ are canonically isomorphic to $\mathrm{H}^{1}\left(\mathcal{O}_{X}\right)$. The pull-back by the absolute Frobenius $F$ of $X$ induces a rational map

$$
F^{*}: \operatorname{PExt}^{1}\left(A_{1}, A_{1}\right) \longrightarrow \operatorname{PExt}^{1}\left(\mathcal{O}_{X}, \mathcal{O}_{X}\right),
$$

which coincides with the projectivized $p$-linear map on the cohomology $\mathrm{H}^{1}\left(\mathcal{O}_{X}\right) \rightarrow$ $\mathrm{H}^{1}\left(\mathcal{O}_{X}\right)$ induced by the Frobenius map $F$. Since we have assumed $X$ ordinary, this $p$-linear map is bijective. Hence we obtain that there is only one (strictly) semistable bundle $E$ such that $[E]=A_{1}$ and $F^{*} E \cong \mathcal{O}_{X}^{2}$, namely $E=A_{1} \oplus A_{1}$. In particular there are no $F^{1}$-trivial rank-2 vector bundles over $X$.

By Proposition 3.1 and using the equations (3.2), we easily obtain that the preimage $V^{-1}\left(A_{1}\right)$ is a projective line $\mathbb{L} \cong \mathbb{P}^{1}$, which passes through the two points

$$
(1: 1: 1: 1) \text { and }(0: 0: 1: 1) \text {. }
$$

We now determine the bundles $E$ satisfying $F^{*} E \cong A_{1} \oplus A_{1}$. Given $E$ with $\left[F^{*} E\right]=$ $A_{1} \in \mathbb{P}^{3}$ we easily establish the equivalence

$$
F^{*} E \cong A_{1} \oplus A_{1} \quad \Longleftrightarrow \quad \operatorname{dim} \operatorname{Hom}\left(F^{*} E, A_{1}\right)=\operatorname{dim} \operatorname{Hom}\left(E, F_{*} A_{1}\right)=2 .
$$

Suppose that $E$ is stable and $F^{*} E \cong A_{1} \oplus A_{1}$. The quadratic map

$$
\text { det }: \operatorname{Hom}\left(E, F_{*} A_{1}\right) \longrightarrow \operatorname{Hom}\left(\operatorname{det} E, \operatorname{det} F_{*} A_{1}\right)=H^{0}\left(\mathcal{O}_{X}(w)\right)
$$


has nontrivial fibre over 0 , since $\operatorname{dim} \operatorname{Hom}\left(E, F_{*} A_{1}\right)=2$. Hence there exists a nonzero $f \in \operatorname{Hom}\left(E, F_{*} A_{1}\right)$ not of maximal rank. We consider the line bundle $N=\operatorname{im} f \subset F_{*} A_{1}$. Since $F_{*} A_{1}$ is stable (see $[\mathrm{LaP}$, Proposition 1.2), we obtain the inequalities

$$
0=\mu(E)<\operatorname{deg} N<\frac{1}{2}=\mu\left(F_{*} A_{1}\right),
$$

a contradiction. Therefore $E$ is strictly semistable and $[E]=\left[A_{2} \oplus A_{2}^{-1}\right]$ for some 4-torsion line bundle $A_{2}$ with $A_{2}^{\otimes 2}=A_{1}$. The $S$-equivalence class $\left[A_{2} \oplus A_{2}^{-1}\right]$ contains three isomorphism classes, and a standard computation shows that only the decomposable bundle $A_{2} \oplus A_{2}^{-1}$ is mapped by $F^{*}$ to $A_{1} \oplus A_{1}$. In particular there are no $F^{2}$-trivial rank-2 bundles.

We now determine the coordinates of $A_{2}$ by intersecting the line $\mathbb{L}$, which can be parametrized by $(r: r: s: s)$ with $r, s \in k$, with the Kummer surface, whose equation is (see [LP2], Proposition 3.1)

$c\left(x_{00}^{2} x_{10}^{2}+x_{01}^{2} x_{11}^{2}\right)+b\left(x_{00}^{2} x_{01}^{2}+x_{10}^{2} x_{11}^{2}\right)+a\left(x_{00}^{2} x_{11}^{2}+x_{10}^{2} x_{01}^{2}\right)+x_{00} x_{01} x_{10} x_{11}=0$.

The computations are straightforward and will be omitted. Let $u \in k$ be a root of the equation

$$
u^{2}+u=b
$$

Then $u+1$ is the other root. The coordinates of the two 4-torsion line bundles (modulo the canonical involution of the Jacobian of $X$ ) $A_{2}$ such that $A_{2}^{\otimes 2}=A_{1}$ are

$$
(u: u: \sqrt{b}: \sqrt{b}) \quad \text { and } \quad(u+1: u+1: \sqrt{b}: \sqrt{b}) .
$$

Now the equation $u=0$ (resp. $u+1=0$ ) implies by (4.3) that $b=0$, which is excluded because we have assumed $X$ smooth. So by Proposition 3.1 the preimage $V^{-1}\left(A_{2}\right)$ consists of the 4 line bundles $A_{3}$ such that $A_{3}^{\otimes 2}=A_{2}$. In particular there are no $F^{3}$-trivial rank-2 bundles.

One easily verifies that the image under the rational map $V$ given by (3.2) of the hyperplane $x_{00}=0$ is the quartic surface given by the equation

$$
b x_{11}^{2} x_{10}^{2}+c x_{11}^{2} x_{01}^{2}+a x_{10}^{2} x_{01}^{2}+x_{00} x_{10} x_{01} x_{11}=0 .
$$

When we replace $\left(x_{00}: x_{01}: x_{10}: x_{11}\right)$ with $(u: u: \sqrt{b}: \sqrt{b})$ in (4.4) we obtain the equation

$$
b^{2}+u^{2}(1+a+c)=0 .
$$

Similarly replacing $\left(x_{00}: x_{01}: x_{10}: x_{11}\right)$ with $(u+1: u+1: \sqrt{b}: \sqrt{b})$ in (4.4) we obtain the equation

$$
b^{2}+\left(u^{2}+1\right)(1+a+c)=0 .
$$

Finally the product of (4.5) with (4.6) equals (here one uses (4.3)) equation (4.1) up to a factor $b^{2}$, which we can drop since $b \neq 0$ - note that we have assumed $X$ smooth, hence $b \neq 0$ by [LP2, Lemma 2.1]. To summarize we have shown that if (4.1) holds, then by Proposition 3.1 there exists an 8-torsion line bundle $A_{3}$ with $A_{3}^{\otimes 4}=A_{1}$ and such that the preimage $V^{-1}\left(A_{3}\right)$ is a projective line $\Delta \subset \mathbb{P}^{3}$.

Consider a point $[E] \in \Delta$ away from the Kummer surface - note that $\Delta$ is not contained in the Kummer surface $\operatorname{Kum}_{X}$ because its intersection is contained in the set of 16 -torsion points. Then $E$ is stable and $\left[F^{*} E\right]=\left[A_{3} \oplus A_{3}^{-1}\right]$. There are three isomorphism classes represented by the $S$-equivalence class $\left[A_{3} \oplus A_{3}^{-1}\right]$, namely the trivial extension $A_{3} \oplus A_{3}^{-1}$ and two nontrivial extensions (for the details 
see [LP1, Remark 6.2]). Since $E$ is invariant under the hyperelliptic involution we obtain $F^{*} E=A_{3} \oplus A_{3}^{-1}$ and finally that $E$ is $F^{4}$-trivial. Hence any stable point on $\Delta$ is $F^{4}$-trivial.

Therefore, assuming (4.1), there exists a 1-dimensional subvariety $\Delta_{0} \subset \mathcal{M}_{X} \backslash$ $\operatorname{Kum}_{X}$ parametrizing all $F^{4}$-trivial rank-2 bundles. Passing to an étale cover $S \rightarrow$ $\Delta_{0}$ ensures existence of a "universal" family $\mathcal{E} \rightarrow X \times S$ and we are done.

Remark. Note that equation (4.1) depends on the choice of a nontrivial 2-torsion line bundle $A_{1}$. If one chooses the 2 -torsion line bundle $(0: 0: 1: 0)$ or $(0: 0: 0: 1)$ (see (4.2)), then the correponding equations are

$$
a^{2}+b^{2}+c^{2}+a+b=0 \quad \text { or } \quad a^{2}+b^{2}+c^{2}+b+c=0 .
$$

\section{REFERENCES}

[LaP] H. Lange, C. Pauly: On Frobenius-destabilized rank-2 vector bundles over curves, arxiv.math.AG/0309456

[LP1] Y. Laszlo, C. Pauly: The action of the Frobenius maps on rank 2 vector bundles in characteristic 2, J. Alg. Geometry 11 (2002), 219-243 MR.1874113 (2003a:14051)

[LP2] Y. Laszlo, C. Pauly: The Frobenius map, rank 2 vector bundles and Kummer's quartic surface in characteristic 2 and 3, Adv. Math. 185, No. 2 (2004), 246-269 MR2060469 (2005d:14049)

[LeP] J. Le Potier: Lectures on vector bundles, Cambridge Studies in Advanced Mathematics 54, Cambridge University Press, 1997 MR.1428426 (98a:14019)

[MS] V.B. Mehta, S. Subramanian: On the fundamental group scheme, Invent. Math. 148 (2002), 143-150 MR 1892846 (2004c:14089)

[N] M.V. Nori, The fundamental group scheme, Proc. Indian Acad. Sci. (Math. Sci.) 91 (1982), 78-122 MR0682517 (85g:14019)

Département de Mathématiques, Université de Montpellier II - Case Courrier 051, Place Eugène Bataillon, 34095 Montpellier Cedex 5, France

E-mail address: pauly@math.univ-montp2.fr 